\title{
EFFECTS OF LESIONS IN THE BASOLATERAL AMYGDALA ON FLUID INTAKE IN THE RAT ${ }^{1}$
}

\author{
BARBARA J. ROLLS ${ }^{2}$ AND EDMUND T. ROLLS \\ Department of Experimental Psychology, University of Oxford
}

\begin{abstract}
Drinking response to hypertonic saline, a cellular thirst stimulus, and to isoproterenol, probably an extracellular thirst stimulus, was normal in rats with bilateral lesions in the basolateral region of the amygdala. The overnight water intake of the lesioned rats was a little higher than normal. However, the lesioned rats showed a major impairment in learning to avoid ingesting a poisonous solution of $\mathrm{LiCl}$ when they were thirsty. The lesioned rats also showed an increased preference of $25 \%$ sucrose in a two-bottle sucrose-water test. The main conclusion is that the basolateral region of the amygdala is involved in the effects of previous experience on drinking and not primarily in the cellular or extracellular controls of drinking.
\end{abstract}

In the preceding article (Rolls \& Rolls, 1973) it is shown that the basolateral region of the amygdala is involved in food selection. It does not appear to play a major role in the amount of food eaten. In this article, the role of the amygdala in the regulation of fluid intake is considered. It has previously been shown that amygdaloid neurons are directly excited in stimulus-bound drinking and eating and have the same refractory periods as the neurons involved in the drinking and eating (Rolls, 1972, 1973).

Lesions in the amygdala can lead to increased or decreased water intake (Grossman \& Grossman, 1963). It is not possible to determine whether there was a specific deficit in the regulation of water intake because tests for osmotic regulation (Fitzsimons, 1961a) and extracellular regulation (Fitzsimons, 1961b; Stricker, 1966) of water intake were not reported. Injections of carbachol into the amygdala do not induce water intake but can modulate drinking induced by water deprivation (Grossman, 1964). Kemble and Schwartzbaum (1969) reported that rats with amygdala lesions drank less of an $8 \%$ sucrose solution than intact rats when given daily access to it for a short time. Monkeys with amygdala

\footnotetext{
${ }^{1}$ Supported in part by the Medical Research Council.

${ }^{2}$ Née Simons. Requests for reprints should be sent to Barbara J. Rolls, Department of Experimental Psychology, University of Oxford, Oxford, England.
}

lesions show a greater preference for strong saccharine solutions than controls (Weiskrantz, 1960).

In the experiments described here, we made lesions in the basolateral region of the amygdala. This is the region in which neurons are activated in electrically induced drinking (Rolls, 1972). We then investigated alterations in fluid intake by measuring daily water intake, and regulation to both osmotic and extracellular stimuli for drinking. We also measured fluid preferences and the effects of previous experience on fluid intake, as it appears that the amygdala is involved in food preferences and the effects of previous experience on food intake (Rolls \& Rolls, 1973).

\section{General Method}

\section{Subjects}

The rats were the same as those used for the experiments described in the preceding article (Rolls \& Rolls, 1973). The animals had bilateral lesions in the lateral amygdaloid region. The majority of the experiments described here were performed after those described in the preceding article. Use of the same animals for the different studies made it possible to determine whether different types of ingestive behavior were influenced by exactly the same lesions.

\section{Procedure}

The rats were maintained in individual cages, and laboratory chow-Dixon's FFG(M)-and drinking water in calibrated columns were continuously available unless otherwise stated. If a rat was water deprived, at least 2 days were allowed before the next test. 
Unless otherwise stated, fluid intakes are given as $M \pm S E_{M}$, and two-tailed $t$ tests are used for statistical comparisons.

\section{EXPERIMENT 1}

In this experiment the overnight water intakes of the amygdala-lesioned and intact rats were compared.

\section{Method}

Procedure. Water was available between 1700 and $0900 \mathrm{hr}$. and the water intake of the lesioned and control rats was measured daily. The lesioned subjects were the 33 rats with amygdala lesions (Am group) described in the preceding article (Rolls \& Rolls, 1973). The postoperative period for which each rat was studied was at least 14 days.

\section{Results}

The mean daily water intakes of the rats are shown with standard deviations in Figure 1. It is clear that following a period of recovery from the operation, the amygdala-lesioned rats' intake was greater than the controls' averaged intake $(p<.01$ on Day $10 ; p<.001$ on Days 15 and 25.) The variance of the intake of the Am rats was greater than that of the controls, as shown by their greater standard deviations

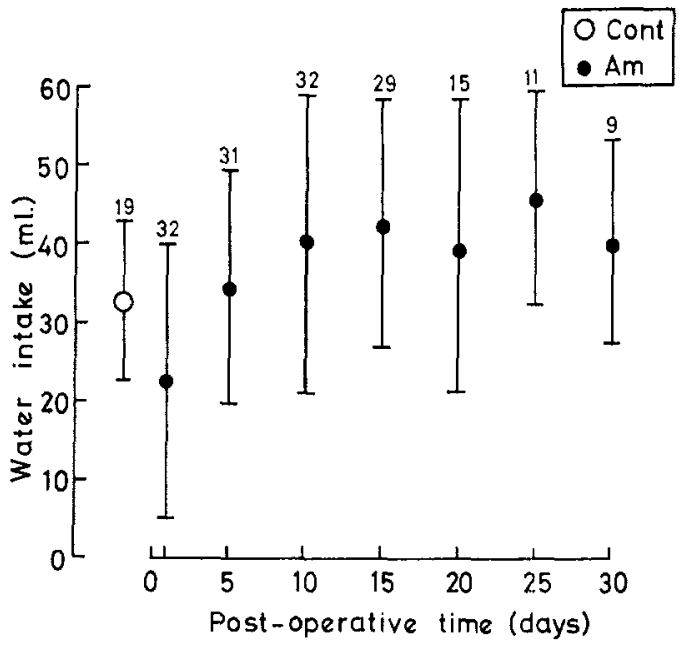

Fig. 1. Overnight water intake $(M \pm S D)$ of the amygdala-lesioned rats. (The rats were lesioned on Day 0. For comparison, the overnight water intake $[M \pm S D]$ of the control rats averaged over the whole period is shown on the left of the abscissa. The number of rats over which the intake was averaged is shown above each point of the graph.) in Figure 1. The variance is significantly greater (two-tailed variance ratio or $F$ test) on Days 1-20 (Day $1, p<.02$; Day $5, p<.05$; Day $10, p<.01 ;$ Day $15, p<$ $.05 ;$ Day $20, p<.01)$. The Am rats with the best lesions in the basolateral region of the amygdala (the BL group of Rolls \& Rolls, 1973) showed a similar increase in the magnitude and variance of the intake.

\section{Discussion}

These results show that the amygdalalesioned rats drank very different amounts of water from each other and on the average drank more water than the controls. This is consistent with the findings of Grossman and Grossman (1963), who found that posterior amygdala lesions tended to increase water intake and anterior amygdala lesions to decrease it. We tested whether the small average increase in the water intake of the lesioned rats was associated with the ingestion of dry food. In the test, the water intakes of the rats were compared on nights when they had and did not have food. When food was not available, the amygdalalesioned rats' intakes fell by $6.81 \pm 2.90$ ml. $(n=29)$ and the control rats' intakes fell by $3.05 \pm 2.25 \mathrm{ml}$. $(n=19)$. This difference is not significant $(t=.94)$. Therefore it was concluded that the slight increase in the water intake of the amygdala-lesioned rats was not associated with the ingestion of dry food. In the next two experiments, more specific investigations into the effects of amygdala lesions on water intake were performed. In Experiment 2 the regulation to an osmotic stimulus was tested, and Experiment 3 was performed to investigate regulation to an extracellular stimulus.

\section{EXPERIMENT 2}

The regulation of amygdala-lesioned and intact rats to an osmotic or cellular stimulus of drinking was measured.

\section{Method}

Procedure. Because the procedure involved nephrectomy, this was the last experiment to be performed on the rats used in Rolls and Rolls (1973). The number of rats left had decreased in the amygdala-lesioned group to 17 , and in the control group 
to 13. The procedure followed that of Fitzsimons (1961a). The rats-in water balance at the start of the experiment-were anesthetized with ether, nephrectomized bilaterally to prevent excretion of the salt load, given an ip injection $(.5 \mathrm{ml} / 100 \mathrm{gm})$ of $2 \mathrm{M} \mathrm{NaCl}$, replaced in their cages, allowed to recover from the ether, and given access to water in a column-but no food-for $6 \mathrm{hr}$. The water intake at the end of the 6-hr. period was measured. The animals were then perfused and the brains prepared for histology as described in the preceding article.

\section{Results}

The water intake of the amygdala-lesioned group was $4.77 \pm .50 \mathrm{ml} / 100 \mathrm{gm}$ and of the control group $5.43 \pm .48 \mathrm{ml} / 100 \mathrm{gm}$. There is no significant difference between these means $(t=.93, p>.3)$. The $S D$ of the intake of the amygdala-lesioned group is $2.05 \mathrm{ml} / 100 \mathrm{gm}$, and of the control group, $1.74 \mathrm{ml} / 100 \mathrm{gm}$. These values are not significantly different.

\section{Discussion}

It is concluded that there is no deficit in the regulation of the drinking response to an osmotic stimulus in these amygdala-lesioned rats.

\section{EXPERIMENT 3}

The drinking response of the amygdalalesioned and intact rats to isoproterenol (isoprenaline) was measured. Isoproterenol is thought to stimulate drinking through the renin-angiotensin system (Houpt \& Epstein, 1971). The renin-angiotensin system appears to mediate the response of rats to at least some extracellular stimuli of thirst (Fitzsimons, 1969; Fitzsimons \& Simons, 1969). Thus, this isoproterenol experiment may measure whether the amygdala-lesioned rats can respond to at least some extracellular stimuli of drinking.

\section{Method}

Procedure. Fourteen amygdala-lesioned and 12 control rats in water balance were lightly anesthetized with ether and given an sc injection of .04 $\mathrm{mg} / \mathrm{kg}$ of isoproterenol (Suscardia, or isoprenaline hydrochloride, Pharmax Ltd.). After recovery from the anesthetic, they were replaced in their cages with a water column but no food available. Water intake was measured over $3 \mathrm{hr}$.

\section{Results}

The water intake of the amygdala-lesioned rats was $1.51 \pm .14 \mathrm{ml} / 100 \mathrm{gm}$, and of the control rats, $1.60 \pm .14 \mathrm{ml} / 100 \mathrm{gm}$. These means are not significantly different $(t=$ $.50, p>.6)$. The $S D \mathrm{~s}(.52$ and .48$)$ are also very similar.

\section{Discussion}

It is concluded that the response to isoproterenol was normal in the amygdala-lesioned group. If isoproterenol acts through the renin-angiotensin system (Houpt \& Epstein, 1971), and this is involved in the drinking response to some extracellular stimuli, then at least this aspect of the extracellular control of water intake was normal in the amygdala-lesioned rats.

No deficits in the osmotic or extracellular controls of drinking in amygdala-lesioned rats were found in Experiments 2 and 3 . This is in line with the rather small average alteration of daily water intake in the group of amygdala-lesioned rats in Experiment 1. The overdrinking and underdrinking in the amygdala-lesioned group, seen in the large standard deviation of the lesioned group's daily intake, remain unexplained. In the next experiments, the effects of amygdala lesions on the nature of the fluids ingested-rather than on the amount of fluid ingested-are investigated.

\section{EXPERIMENT 4}

The purpose of the experiment was to determine whether rats with amygdala lesions choose an osmotically appropriate fluid to drink after water deprivation. Rats were given a choice between $25 \%$ sucrose, which is hypertonic, and water.

\section{Method}

Procedure. Thirty-one rats with amygdala lesions (including all but two of the Am group of the preceding article and all but one of the rats in the BL group) and 22 control rats (the original control group and three additional rats) were the subjects. They were water- but not food-deprived overnight. Then water and $25 \%(\mathrm{w} / \mathrm{v})$ sucrose solution in columns were placed on the individual cages in a two-bottle choice situation. To control for position preferences, the experiment was repeated twice in a counterbalanced design with the 
water either on the left or on the right. The amounts of water and sucrose solution consumed were measured after $1 \mathrm{hr}$. and $6 \mathrm{hr}$. The amounts of the fluids consumed per $100 \mathrm{gm}$. of initial body weight were averaged for each rat over the left and right positions. The mean intake per 100 gm. of each group at the different times was then calculated. No food was available during the tests.

\section{Results}

The mean intake of each group at different times after access to the two fluids is shown in Figure 2. After $1 \mathrm{hr}$. the BL group had ingested more $25 \%$ sucrose than the controls $(p<.05)$. After $6 \mathrm{hr}$. the BL group had ingested $5.20 \pm .49 / 100 \mathrm{gm}$ and the Am group $4.79 \pm .26 \mathrm{ml} / 100 \mathrm{gm}$ of sucrose, while the control group had ingested only $3.54 \pm .33 \mathrm{ml} / 100 \mathrm{gm}$ of sucrose. Both lesioned groups drank significantly more sucrose than the control rats $(p<.01)$. Although after $6 \mathrm{hr}$. the lesioned groups drank less water than the controls, the differences were not significant. Thus, the lesioned rats drank similar amounts of water to those of the controls, but after $6 \mathrm{hr}$. they had ingested significantly more $25 \%$ sucrose solution than the controls. This difference is more extreme in the $\mathrm{BL}$ group and be-

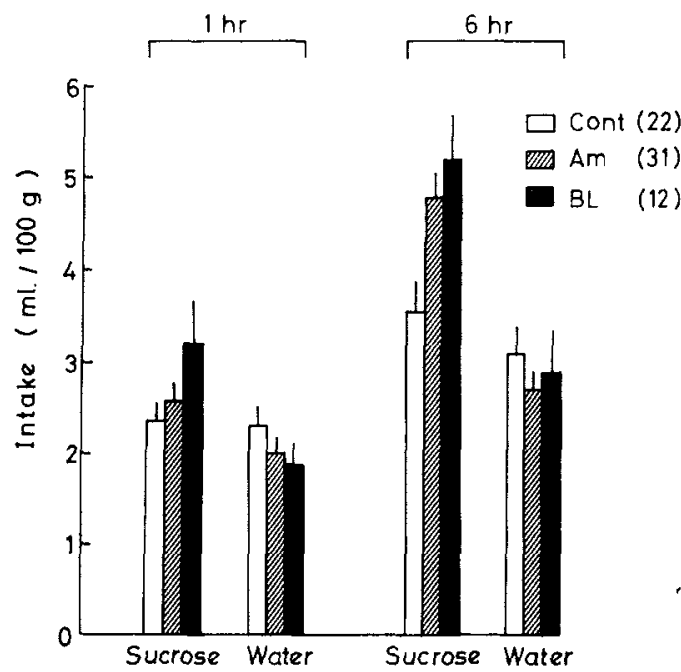

Fia. 2. The intake $(M \pm S E)$ of $25 \%$ sucrose solution and of water by the control and amygdala-lesioned rats ( $\mathrm{Am}$ and $\mathrm{BL}$ groups) after 1 and $6 \mathrm{hr}$. in a two-bottle preference test. (The number of rats in each group is shown in parentheses.) comes significant $(p<.05)$ during the first 1-hr. drinking period for this group.

\section{Discussion}

The abnormally large intake of $25 \%$ sucrose by the amygdala-lesioned group could be due to failure of the homeostatic signal to restrict intake of the hypertonic sucrose in the test or to an increased reactivity to sucrose. As the response to an osmotic stimulus is normal in these rats (Experiment 2), failure to respond appropriately to a homeostatic signal seems an unlikely explanation of the present results. It may therefore be that the rats are hyperreactive to the taste of the sucrose solution. This explanation would be in line with results in monkeys, which drink more strong saccharine solution after amygdalectomy (Weiskrantz, 1960). The saccharine would provide a taste but not an osmotic or metabolic signal.

\section{EXPERIMENT 5}

Altered food preferences (preceding article) and altered ingestion of fluids (Experiments 4 and 6 ) were found in the amygdalalesioned rats. It is therefore of interest to determine whether these rats underreact to $8 \%$ sucrose solution, as this has been reported to follow large lesions in the amygdala (Kemble \& Schwartzbaum, 1969). This is the experiment described here.

\section{Method}

Procedure. Twenty rats with amygdala lesions and 11 controls were in food and water balance at the start of the experiment. The experiment was conducted in the morning. A column of $8 \%$ sucrose solution $(w / v)$ was placed on each rat's cage, and the amount consumed in $30 \mathrm{~min}$. was measured.

\section{Results}

The amygdala-lesioned group ingested $1.76 \pm .33 \mathrm{ml} / 100 \mathrm{gm}$ of the $8 \%$ sucrose and the control group ingested $1.45 \pm .18 \mathrm{ml} / 100$ $\mathrm{gm}$. The difference is not significant $(t=$ $.651, p>.5$ ). One rat in the Am group did not drink in the 30-min. period.

\section{Discussion}

In Kemble and Schwartzbaum's (1969) experiment, the rats with amygdala lesions 
drank less $8 \%$ sucrose than the control rats. They concluded that their lesioned rats underreacted to the sucrose solution. The rats with amygdala lesions in the present study did not drink significantly differently from the controls, although the mean for the amygdala-lesioned group was greater than that of the controls. It is concluded that either the lesions in Kemble and Schwartzbaum's study were in a different region of the amygdala to those in this study, or that their slightly different procedure might have enabled them to find a difference.

\section{EXPERIMENT 6}

The rats with amygdala lesions were not impaired on thirst tests (Experiments 2 and 3 ) and reacted normally to $8 \%$ sucrose (Experiment 5), but when thirsty drank more $25 \%$ sucrose than water in a twobottle preference test (Experiment 4). Their choice of $25 \%$ sucrose in Experiment 4 may not be guided normally by homeostatic necessity. To determine whether the fluid ingestion of amygdala-lesioned rats is guided by experience, a "learned aversion" experiment was performed. Normal rats poisoned by the ingestion of $\mathrm{LiCl}$ subsequently avoid $\mathrm{LiCl}$ and $\mathrm{NaCl}$ (Nachman, 1963). In this type of learning, the experience of ingesting $\mathrm{LiCl}$ and feeling ill comes to guide subsequent behavior.

\section{Method}

Procedure. Ten of the amygdala-lesioned and 16 of the control rats were the subjects in this experiment. On Day 1 the rats were put on a $23-\mathrm{hr}$. water-deprivation schedule with chow continuously available. On Day 4, $12 \mathrm{M} \mathrm{LiCl}$ instead of water was available in the drinking column. The columns were removed when the rats had drunk $8 \mathrm{ml}$. or after $15 \mathrm{~min}$., whichever was sooner. Because some of the amygdala-lesioned rats drank only small volumes of the $\mathrm{LiCl}$ solution, the intakes of six of the control rats were restricted to $2-3 \mathrm{ml}$. (see Figure 3). The 8-ml. value was chosen so that the sickness should not be severe, and no sign of sickness was seen. Nachman's (1963) albino rats showed sickness by lying down on the floors of their cages, but they consumed an average of $9 \mathrm{ml}$. of $.12 \mathrm{M} \mathrm{LiCl}$ solution. Food and water were available ad lib for the night of Day 4 and for Days 5 and 6 . On Night 6 the rats were water deprived, and on Day 7 the drinking response to $.12 \mathrm{M} \mathrm{NaCl}$ was measured. At the end of $30 \mathrm{~min}$. the amount drunk was noted and the experiment was termi- nated. The $\mathrm{NaCl}$ solution was used because rats react to it as to $\mathrm{LiCl}$ when tested for learned aversion, yet the $\mathrm{NaCl}$ does not make the rats sick a second time (Nachman, 1963).

\section{Results}

It is shown in Figure 3 that in general the amygdala-lesioned rats drank the $\mathrm{NaCl}$ solution when tested for the learned aversion and the control rats did not. If the animals which drank $6 \mathrm{ml}$. or less of $\mathrm{LiCl}$ are considered, the 7 amygdala-lesioned rats drank significantly more $\mathrm{NaCl}$ than the 11 controls $(p<.001 ;$ Mann-Whitney $U$ test, one-tailed). It is clear from Figure 3 that all these Am rats drank the $\mathrm{NaCl}$ and failed to show learned aversion and that all but one of the control rats drank almost no $\mathrm{NaCl}$ and showed learned aversion.

Three of the rats in the Am group drank more than $6 \mathrm{ml}$. of $\mathrm{LiCl}$ and subsequently drank no $\mathrm{NaCl}$. It appears that the intake of the poisonous $\mathrm{LiCl}$ necessary to produce learned aversion in these animals is at least three times that which is effective in the control rats (see Figure 3 ).

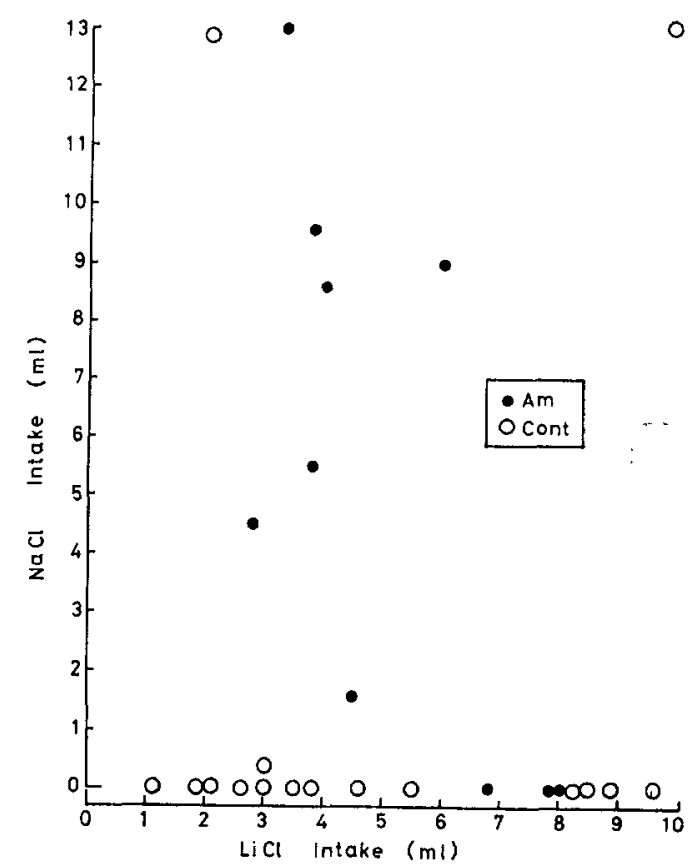

Fia. 3. The $\mathrm{NaCl}$ intake on the second test as a function of $\mathrm{LiCl}$ intake on a first test in a learned-aversion experiment. 


\section{Discussion}

In this experiment it was found that in the Am rats the ingestion of a poison had little effect on the subsequent intake of a solution like the poison. The control of fluid intake by previous experience appeared to be deficient in the lesioned rats. With large $\mathrm{LiCl}$ intakes, learned aversion was apparent; and thus, learning was not completely impossible in the lesioned animals.

Although it is possible that the amygdala lesions resulted in less sickness after ingestion of $\mathrm{LiCl}$, it appears most likely that it was learning which was impaired in the situation (see also General Discussion section).

\section{General Discussion}

The small average increase in the daily water intake of the Am group of rats and the large variance in the water intake of the group (Experiment 1) are consistent with the findings of Grossman and Grossman (1963). They found that posterior amygdala lesions increased water intake, and anterior amygdala lesions decreased it. The rats which died as a result of amygdala lesions (see preceding article) were adipsic, and had lesions in the medial region of the amygdala (see Rolls \& Rolls, 1973; Figure 1). Because osmotic regulation and regulation to at least one extracellular thirst stimulus were found to be normal (Experiments 2 and 3 ) in the Am group, it is concluded that lesions in the basolateral region of the amygdala in the rat probably do not produce a specific defect in the control of water intake.

The rats with amygdala lesions drank significantly more $25 \%$ sucrose solution than control rats in a similar two-bottle situation with a choice between the sucrose solution and water (Experiment 4 ). The $25 \%$ sucrose solution is hypertonic. Thus the increased preference for the sucrose is probably an osmotically inappropriate choice. What may be homeostatically appropriate did not control choice in this situation, perhaps because the rats had a greater affective reaction to the sucrose or because the rats were not able to terminate behavior which had immediate pleasant effects (taste) but delayed aversive effects (hypertonicity). Relative to controls, monkeys with medial temporal lesions which include the amygdala also show an increased preference for strong sweet solutions. For example, with a choice between $.25 \%$ saccharine and water, the lesioned monkeys drank relatively much more saccharine than water, and one monkey consumed an average of $3,550 \mathrm{ml}$. of saccharine a day, which was almost equal to his body weight (Weiskrantz, 1960). In a one-bottle test with $8 \%$ sucrose (Experiment 5), the Am rats drank more sucrose than the controls, but the difference was not significant. The increased preference is in line with the results of Experiment 4, but is different from that found in Kemble and Schwartzbaum's (1969) group of rats with amygdala lesions.

The finding that the amygdala-lesioned rats did not show learned aversion (Experiment 6) shows one clear way in which the amygdala is involved in fluid intake. Signals which inhibit fluid intake as a result of previous experience do not appear to operate after the lesions in the basolateral amygdala. This conclusion is similar to that reached in the preceding paper (Rolls \& Rolls, 1973), that the basolateral amygdala is concerned with the selection of foods on the basis of previous experience.

This modulation of food and water intake on the basis of previous experience could operate by pathways connecting the amygdala to the hypothalamus. These pathways would normally carry the signals indicating that eating or drinking should proceed or that eating or drinking should stop. Activation of these pathways might therefore be a factor in eating and drinking elicited by hypothalamic stimulation in that a signal capable of controlling eating and drinking is carried by these pathways. Amygdalo-hypothalamic pathways are activated in eating and drinking elicited by lateral hypothalamic stimulation (Rolls, 1972). As the absolute refractory period of neurons in this pathway is similar to that of neurons involved in the eating and drinking elicited by the lateral hypothalamic stimulation, these pathways are probably 
involved in the elicited eating and drinking (Rolls, 1973). Activation of other pathways may also be necessary for stimulus-bound motivational behavior. For example, neurons in the midbrain and pons are excited by lateral hypothalamic stimulation, and through these neurons arousal is produced (Rolls, 1971a). Stimulation of the nucleus accumbens does not excite these brainstem neurons and probably does not lead to stimulus-bound motivational behavior (Rolls, $1971 b)$. Thus the excitation of the brainstem neurons may be necessary for stimulusbound motivational behavior. Stimulusbound eating and drinking may occur when amygdalo-hypothalamic pathways which can control eating and drinking are activated by hypothalamic stimulation which also produces arousal by activating brainstem neurons.

The amygdalo-hypothalamic pathways could modulate ingestion by making it rewarding or aversive (cf. Cabanac, 1971). If the pathways can modulate reward level, then this could account in part for reward produced by hypothalamic stimulation. Amygdalo-hypothalamic pathways are activated in reward produced by lateral hypothalamic stimulation (Rolls, 1972).

The rats with basolateral amygdala lesions described in this and the preceding paper did not inhibit their food and fluid intake normally on the basis of previous experience. The deficits were in specific feeding and drinking situations. The learned aversion experiment is specifically related to ingestion in that an animal must associate the ingestion of a particular substance with the aversive consequences of the ingestion which may be considerably delayed. A similar delay does not occur in the standard passive-avoidance experiment, in which an animal learns not to ingest a substance if a shock occurs at ingestion. Rats with amygdala lesions are impaired additionally in tasks not related specifically to food or water intake. They do not form a conditioned emotional response to pairing of light with shock (i.e., response suppression does not occur) and do not inhibit responses in the nonreinforced parts of a discrimination task (Thompson \& Schwartzbaum, 1964). They also perform poorly in shock-avoidance tasks (Robinson, 1963). A common explanation could exist for these findings and the deficits in feeding and drinking described here. For example, both classes of situation may involve a failure of inhibition or a failure to respond to aversive stimuli. But this type of explanation may be unsatisfactory, because the feeding and drinking deficits were found after basolateral amygdala lesions and the other deficits after lesions in the cortico-medial region of the amygdala.

Our conclusion that the basolateral region of the amygdala is involved in the effects of previous experience on food and fluid intake is not intended to exclude other, perhaps more general, interpretations of the function of the amygdala. But the present findings that the amygdala does influence food (see Rolls \& Rolls, 1973) and fluid intake suggests that functional connections exist between the amygdala and the centers which control feeding and drinking. This suggestion is consistent with the discussion above on the function of the amygdalohypothalamic connections.

The results of these and other experiments suggest that one role of the amygdala in ingestive behavior is as follows. The basolateral amygdala inhibits eating and drinking under conditions determined by previous experience. As shown here, these conditions include previous experience with a food (reducing neophobia) and the effects of a poison. One stage in the pathway through which this type of effect is mediated appears to be the lateral hypothalamus, for rats with lateral hypothalamic lesions show an impairment in learning to avoid food poisoned with $\mathrm{LiCl}$. (This experiment was performed on recovered lateral hypothalamic rats by Roth, Schwartz, \& Teitelbaum; a description of the experiments appeared in Teitelbaum, 1971.) Pathways from the amygdala to the hypothalamus through which the inhibition could be mediated have been described anatomically and neurophysiologically (e.g., Dreifuss, 1972; Egger, 1972; Lammers, 1972; Murphy, 1972; Rolls, 1972; Van Atta \& Sutin, 1972). The necessary inputs to the amygdala would appear to be incoming sensory information and in- 
formation from memory about the consequences of previous ingestion of the food. A function of the basolateral amygdala could be to produce the inhibitory signal from these inputs.

\section{REFERENCES}

Cabanac, M. Physiological role of pleasure. Science, 1971, 173, 1103-1107.

Dreifuss, J. J. Effects of electrical stimulation of the amygdaloid complex on the ventromedial hypothalamus. In B. E. Eleftheriou (Ed.), The neurobiology of the amygdala. New York: Plenum, 1972.

EGGER, M. D. Amygdaloid-hypothalamic neurophysiological interrelationships. In B. E. Eleftheriou (Ed.), The neurobiology of the amygdala. New York: Plenum, 1972.

Fitzsimons, J. T. Drinking by nephrectomized rats injected with various substances. Journal of Physiology, 1961, 155, 563-579. (a)

Fitzimons, J. T. Drinking by rats depleted of body fluid without increase in osmotic pressure. Journal of Physiology, 1961, 159, 297-309. (b)

Fitzsimons, J. T. The role of a renal thirst factor in drinking induced by extracellular stimuli. Journal of Physiology, 1969, 201, 349-368.

Fitzisimons, J. T., \& Simons, B. J. The effect on drinking in the rat of intravenous infusion of angiotensin, given alone or in combination with other stimuli of thirst. Journal of Physiology, 1969, 203, 45-57.

Grossman, S. P. Behavioral effects of chemical stimulation of the ventral amygdala. Journal of Comparative and Physiological Psychology, 1964, 57, 29-36.

Grossman, S. P., \& Grossman, L. Food and water intake following lesions or electrical stimulation of the amygdala. American Journal of Physiology, 1963, 205, 761-765.

Houpt, K. A., \& Epstein, A. N. The complete dependence of beta-adrenergic drinking on the renal dipsogen. Physiology and Behavior, 1971, 7, 897-902.

Kemble, E. D., \& Schwartzbaum, J. S. Reactivity to taste properties of solutions following amygdaloid lesions. Physiology and Behavior, 1969, 4, 981-985.

Lammers, H. J. The neural connections of the amygdaloid complex in mammals. In B. E. Eleftheriou (Ed.), The neurobiology of the amygdala. New York: Plenum, 1972.
Murphy, J. T. The role of the amygdala in controlling hypothalamic output. In B. E. Eleftheriou (Ed.), The neurobiology of the amygdala. New York: Plenum, 1972.

Nachman, M. Learned aversion to the taste of lithium chloride and generalization to other salts. Journal of Comparative and Physiological Psychology, 1963, 56, 343-349.

RoBINSON, E. Effect of amygdalcctomy on fear-motivated behavior in rats. Journal of Comparative and Physiological Psychology, 1963, 56, 814-820.

RoLls, E. T. Contrasting effects of hypothalamic and nucleus accumbens septi self-stimulation on brain stem single unit activity and cortical arousal. Brain Research, 1971, 31, 275-285. (a)

Rolls, E. T. Involvement of brainstem units in medial forebrain bundle self-stimulation. Physiology and Behavior, 1971, 7, 297-310. (b)

RoLLs, E. T. Activation of amygdaloid neurones in reward, eating and drinking elicited by electrical stimulation of the brain. Brain Research, $1972,45,365-381$.

RoLLS, E. T. Refractory periods of neurons directly excited in stimulus-bound eating and drinking in the rat. Journal of Comparative and Physiological Psychology, 1973, 82, 15-22.

Rolls, E. T., \& Rolls, B. J. Altered food preferences after lesions in the basolateral region of the amygdala in the rat. Journal of Comparative and Physiological Psychology, 1973, 83, 248-259.

Stricker, E. M. Extracellular fluid volume and thirst. American Journal of Physiology, 1966, 211, 232-238.

Teitelbaum, $P$. The encephalization of hunger. In E. Stellar \& J. M. Sprague (Eds.), Progress in physiological psychology. Vol. 4. New York: Academic Press, 1971.

Thompson, J. B., \& Schinartzbaum, J. S. Discrimination behavior and conditioned suppression (CER) following localized lesions in the amygdala and putamen. Psychological Reports, $1964,15,587-606$.

Van AtTa, L., \& Sutin, J. Relationships among amygdaloid and other limbic structures in influencing activity of lateral hypothalamic neurons. In B. E. Eleftheriou (Ed.), The neurobiology of the amygdala. New York: Plenum, 1972.

Weiskrantz, L. Effects of medial temporal lesions on taste preference in the monkey. Nature, $1960,187,879-880$.

(Received May 1, 1972) 